\title{
Serum concentrations of vascular endothelial growth factor in relation to retinopathy of prematurity
}

\author{
Gunnel Hellgren 1,2, Chatarina Löfqvist ${ }^{2}$, Anna-Lena Hård², Ingrid Hansen-Pupp ${ }^{3}$, Magnus Gram4, David Ley ${ }^{3}$, Lois E Smith ${ }^{5}$ and \\ Ann Hellström²
}

BACKGROUND: The role of vascular endothelial growth factor (VEGF) in the pathogenesis of retinopathy of prematurity (ROP) has been clearly established. However, little is known about temporal changes in circulating VEGF concentrations in the preterm infant. The objective was to determine the longitudinal serum concentrations of VEGF in relation to ROP.

METHODS: This study included 52 infants born at $<31$ wk gestational age (non-ROP $n=33$, nonproliferative ROP $n=10$, treated for ROP $n=9$ ). VEGF concentrations were analyzed in blood samples collected at birth, at $3 \mathrm{~d}$ postnatal age, and then weekly until at least a gestational age of $35 \mathrm{wk}$.

RESULTS: VEGF concentrations at birth did not differ between groups, independent of later ROP status. In contrast, VEGF serum concentrations were significantly higher at first detection of ROP in infants who were later treated for ROP compared to infants without ROP. At the time of laser therapy, serum VEGF concentrations did not differ between groups.

CONCLUSION: Circulatory concentrations of VEGF, in infants who later developed severe ROP, were elevated at the time when ROP first was detected but not at the time when current treatment most often occurred. This supports the need for further studies of circulating VEGF in relation to the timing of ROP treatment.

$\mathbf{R}^{\mathrm{e}}$ etinopathy of prematurity (ROP) is a sight-threatening neovascular retinal disease affecting very preterm infants. The complex pathogenesis behind ROP occurs in two phases: in the first phase, vessel growth ceases resulting in ischemia and in the second stage, vascular proliferation occurs. The retinal vascular network begins to develop around the 14th wk of gestation and is fully vascularized around $40 \mathrm{wk}$ of gestation (1). Thus, the lower the GA at birth, the less vascularized the retina and the higher the risk for poor retinal vascular growth and therefore the higher the risk of proliferative ROP.

The pathology of ROP has been studied in animal models and in humans (2-4). Phase I (delayed growth and destruction of already formed retinal vessels) is associated with hyperoxia and suppression of oxygen-regulated growth factors such as vascular endothelial growth factor (VEGF) and loss of growth factors normally provided by the in utero environment. In the second phase, the peripheral now avascular retina matures, but with increased metabolic demand and no blood supply, there is retinal hypoxia which drives increased expression of oxygen-regulated growth factors such as VEGF, resulting in uncontrolled neovascularization (5). In neonates, proliferative ROP usually occurs from 31 to 44 wk of PMA (6). Several risk factors for ROP have been identified (2-4), including unregulated oxygen supplementation (which suppresses normal vascular growth) and low birth weight and GA (resulting in a very unvascularized retina as a starting point) (4). The role of VEGF in the pathogenesis of ROP has been clearly established $(7,8)$.

Little is known regarding local and systemic VEGF concentrations in the preterm neonate. Prior studies examining VEGF concentrations at birth have detected no difference in VEGF concentrations in cord blood between preterm and term infants (9), or between preterm infants who later developed ROP and those who did not develop this disease (10). In addition, no significant differences in VEGF concentrations were found over time or between infants with or without later development of ROP (11). Higher concentrations of VEGF were found in cord blood serum and in serum from neonates compared to concentrations found in adults (12).

Local VEGF concentrations measured in the vitreous and subretinal fluid of eyes undergoing surgery for retinal detachment at late stages of ROP were elevated in ROP eyes compared to eyes undergoing surgery for congenital cataract or eyes with acute retinal detachment (13-15), and VEGF concentrations correlated with vascular activity (defined as dilatation and tortuosity of the posterior pole retinal vessels and neovascularization) $(13,14)$. Decreased concentrations of serum VEGF were found after both local laser and intraocular anti-VEGF treatment for ROP; however, the decrease in VEGF concentrations were less pronounced after laser treatment (16).

The aims of this study were to analyze longitudinal concentrations of serum VEGF in preterm infants and to relate VEGF concentrations to ROP status. 
Table 1. Demographic and morbidity data

\begin{tabular}{|c|c|c|c|c|c|}
\hline & All & No ROP & Nonproliferative ROP & Treated for ROP & $P$ value \\
\hline Number & 52 & 33 & 10 & 9 & \\
\hline GA in weeks, Md (min; max) & $26.5(23.0 ; 30.6)$ & $27.4(24.3 ; 30.6)$ & $25.1(23.4 ; 26.4)$ & $24.1(23.0 ; 27.1)$ & $<0.001$ \\
\hline AGA/SGA, $n(\%)$ & $39 / 13(75 / 25)$ & $29 / 4(88 / 12)$ & $4 / 6(40 / 60)$ & $6 / 3(67 / 33)$ & $<0.01^{\text {a }}$ \\
\hline Single/twin, $n(\%)$ & $27 / 25(52 / 48)$ & $16 / 17(48 / 52)$ & $7 / 3(70 / 30)$ & $4 / 5(44 / 56)$ & $\mathrm{NS}^{\mathrm{a}}$ \\
\hline Birth weight $_{\mathrm{SD}^{\prime}} \mathrm{Md}(\min ; \max )$ & $-0.78(-5.00 ; 0.89)$ & $-0.69(-4.66 ; 0.89)$ & $-2.30(-5.00 ; 0.60)$ & $-0.72(-3.16 ; 0.39)$ & NS \\
\hline BPD, $n(\%)$ & $38(73)$ & $22(67)$ & $9(90)$ & $7(78)$ & $\mathrm{NS}^{\mathrm{a}}$ \\
\hline $\mathrm{NEC}, n(\%)$ & $1(1.9)$ & $0(0)$ & $1(10)$ & $0(0)$ & $\mathrm{NS}^{\mathrm{a}}$ \\
\hline IVH, $n(\%)$ & $14(27)$ & $8(24)$ & $2(20)$ & $4(44)$ & $\mathrm{NS}^{\mathrm{a}}$ \\
\hline PDA, $n(\%)$ & $11(21)$ & $2(6.1)$ & $4(40)$ & $5(56)$ & $0.001^{\mathrm{a}}$ \\
\hline
\end{tabular}

AGA, appropriate for gestational age; BPD, bronchopulmonary dysplasia; $\mathrm{HC}$, head circumference; IVH, intraventricular hemorrhage; Md, median; NEC, necrotizing enterocolitis; PDA, patent ductus arteriosus; ROP, retinopathy of prematurity; SDS, SD score; SGA, small for gestational age.

a tatistical analyses across the three groups were performed with the chi-squared test for frequencies.

\section{RESULTS}

Clinical data at birth and morbidity data of the study population are shown in Table 1.

\section{VEGF Concentrations in Cord Blood}

At birth, VEGF plasma concentrations in cord blood did not correlate with GA, weight, or weight standard deviation score ( weight $_{\mathrm{SDS}}$ ). In addition, plasma concentrations of VEGF in cord blood were analyzed for correlations with later ROP development (no ROP, nonproliferative ROP, or ROP requiring treatment). No correlations were found between VEGF concentrations at birth and later ROP status; median (95\% CI for median) concentrations in cord blood were $20.2(7.2-36.1)$ $\mathrm{pg} / \mathrm{ml}, 32.4(2.1-73.1) \mathrm{pg} / \mathrm{ml}$, and $29.6(6.2-89.8) \mathrm{pg} / \mathrm{ml}$ in infants with no ROP, nonproliferative ROP, and treated for ROP, respectively (Figure 1).

\section{Temporal Changes in VEGF Concentrations in Serum}

Longitudinal serum concentrations of VEGF from 27 to 40 wk PMA according to PMA and ROP status are shown in Figure 2. Median (95\% CI for median) VEGF concentrations are presented for each separate week from 23 to $40 \mathrm{wk}$ PMA in Table 2. Infants requiring treatment for ROP had significantly higher median VEGF serum concentrations at 34, 35, and 36 wk PMA than infants without ROP. Differences between infants with no ROP and those requiring ROP treatment remained significant at PMA 34 and $35 \mathrm{wk}$ after adjustment for GA at birth. Cohen's effect values were $d=-0.35, d=$ -0.44 , and $d=-0.39$ at PMA 34,35 , and 36 weeks, respectively, suggesting a moderate practical significance. Median (range) PMA at first diagnosis of early proliferative ROP was 34 (32$35)$ wk, 35 (33-38) wk when ROP stage 3 was identified, and 39 (34-40) wk at the time of laser therapy (Figure 2). These results indicate significantly elevated VEGF serum concentrations in infants requiring treatment for severe proliferative ROP compared to infants without any ROP, from the first sign

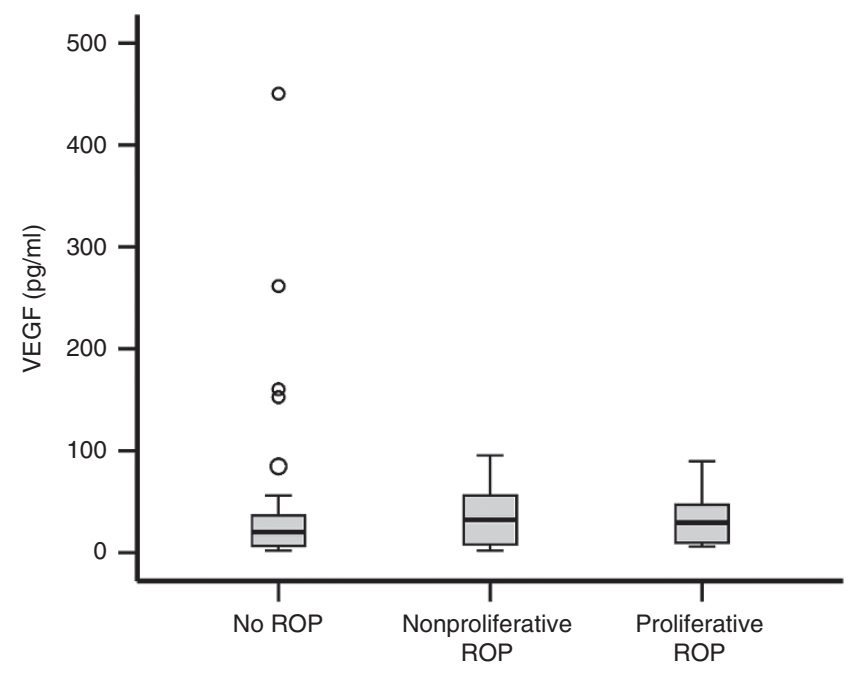

Figure 1. VEGF concentration in cord blood in infants without ROP, with nonproliferative ROP, and infants with proliferative ROP and later treated for severe ROP. There were no significant differences according to ROP status. Open circles represent values classified as outliers. ROP, retinopathy of prematurity; VEGF, vascular endothelial growth factor.

of ROP up to the time point just before ROP laser therapy, but not at the time of laser therapy.

In Figure 3, longitudinal VEGF serum concentrations for the nine infants treated for ROP are shown. Seven infants showed elevated VEGF serum concentrations prior to or at the time when ROP first was diagnosed (A, B, C, D, E, F, and $\mathrm{G})$. One infant $(\mathrm{H})$ did not follow this pattern. Instead VEGF concentrations were low prior to treatment, and maximum VEGF concentration was found after treatment. This child was born at GA $24 \mathrm{wk}$ and had the lowest birth weight ${ }_{\mathrm{SDS}}$ $(-3.16)$ of all treated infants. For one infant (I), ROP screening data until treatment, and serum samples between PMA 31 and $37 \mathrm{wk}$ were missing, leaving eight individuals for evaluation. 


\section{DISCUSSION}

In this study, we investigated the longitudinal concentrations of serum VEGF in extremely preterm infants with respect to the development of ROP. At birth and in early postnatal life,

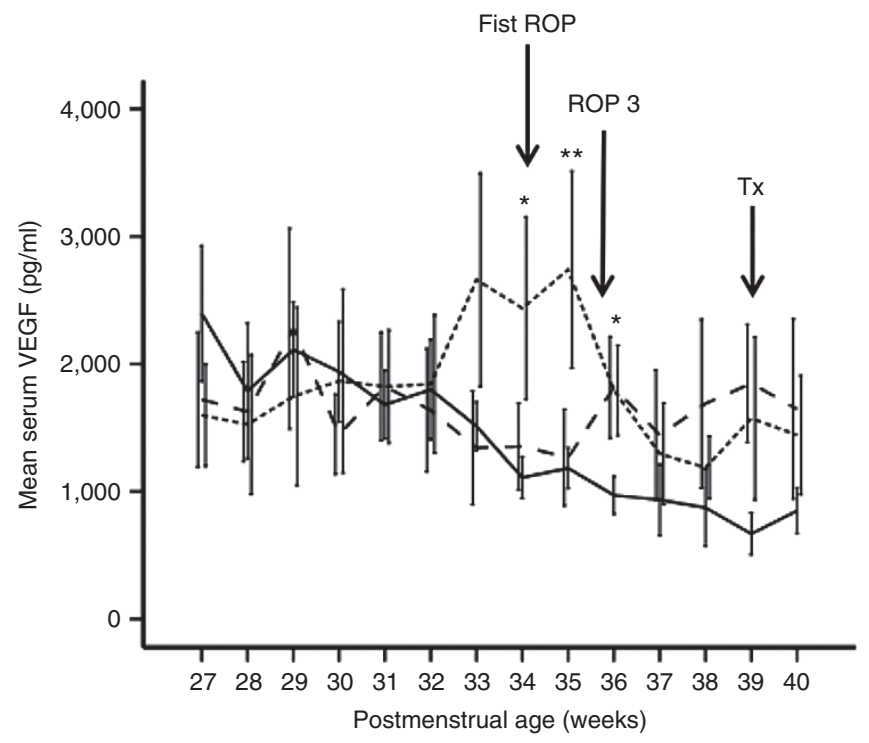

Figure 2. Mean $( \pm S E)$ VEGF concentrations in infants without ROP (solid line), with nonproliferative ROP (dashed line), and treated for ROP (dotted line). Arrows indicate the median time of the first sign of ROP (ROP), when stage 3 ROP was detected (ROP 3), and the time of laser therapy (Tx). ${ }^{*} P$ $<0.05$ treated for ROP vs. infants without ROP, ${ }^{* * P}<0.01$ treated for ROP vs. infants without ROP. ROP, retinopathy of prematurity; VEGF, vascular endothelial growth factor.
VEGF serum concentrations varied broadly and were not correlated with later ROP status. However, at first ROP diagnosis, VEGF serum concentrations were significantly higher in infants who were later treated for severe proliferative ROP. In contrast, at the time of laser treatment, serum VEGF concentrations were the same in infants with and without ROP. These results provide information about systemic longitudinal VEGF concentrations in relation to ROP development.

To our knowledge, this is the first study in which serum VEGF concentrations have been followed in very preterm infants, from birth to PMA $40 \mathrm{wk}$, and related to ROP development. Currently, data on the natural temporal pattern of circulating VEGF after preterm birth is lacking. The results are important, given that during fetal life, VEGF, which is critical in normal angiogenesis, is expressed in most tissues and that decreased concentrations of circulating VEGF have been found, both after the conventional laser treatment and after the recently introduced off-label use of anti-VEGF treatment for ROP (16-18). In these previously reported studies, concentrations were persistently low for at least $60 \mathrm{~d}$ after treatment, independent of the method used; however, Kong et al. (16) reported that the decrease in VEGF serum concentrations after laser treatment was about half the decrease after anti-VEGF treatment.

Although VEGF serum concentrations in this study were highly variable between study subjects, group mean concentrations were higher in infants treated for ROP, at the time when ROP was first diagnosed, than at corresponding time in infants without any stage of ROP. In addition, individual VEGF data, for

Table 2. Median VEGF serum levels by PMA in infants with no ROP $(n=33)$, nonproliferative ROP $(n=10)$, and treated for ROP $(n=9)$

\begin{tabular}{|c|c|c|c|c|c|c|c|c|}
\hline $\begin{array}{l}\text { PMA } \\
\text { (weeks) }\end{array}$ & No ROP & $n$ & Nonproliferative ROP & $n$ & Treated for ROP & $n$ & $\begin{array}{l}P \text { value non-ROP } \\
\text { vs. treated for ROP }\end{array}$ & Effect size \\
\hline 23 & - & - & 1,667 & 1 & 1,375 & 1 & - & - \\
\hline 25 & $1,602(69-2,675)$ & 6 & $1,246(256-2,133)$ & 9 & $1,067(347-4,395)$ & 6 & 1.000 & 0.00 \\
\hline 26 & $412(223-1,012)$ & 12 & $1,057(123-1,944)$ & 11 & $915(338-8,568)$ & 7 & 0.176 & -0.31 \\
\hline 29 & $1,290(720-2,516)$ & 28 & $1,375(467-5,489)$ & 9 & $1,319(87-6,087)$ & 8 & 0.594 & -0.09 \\
\hline 30 & $1,273(422-2,760)$ & 24 & $1,180(294-3,103)$ & 8 & $1,583(262-4,504)$ & 5 & 0.686 & -0.07 \\
\hline 31 & $1,424(464-2,221)$ & 30 & $973(765-3,005)$ & 9 & $2,021(328-3,459)$ & 8 & 0.452 & -0.12 \\
\hline 32 & $1,206(595-2,299)$ & 27 & $1,237(239-4,024)$ & 8 & $1,687(508-4,587)$ & 7 & 0.624 & -0.08 \\
\hline 36 & $817(422-1,390)$ & 21 & $1,266(802-3,084)$ & 9 & $1,835(700-3,574)$ & 7 & 0.041 & -0.39 \\
\hline 37 & $605(58-2,540)$ & 11 & $928(285-3,841)$ & 7 & $1,261(252-2,657)$ & 5 & 0.282 & -0.27 \\
\hline 38 & $581(240-1,923)$ & 5 & $1,532(136-3,913)$ & 5 & $1,352(94-1,750)$ & 6 & 0.465 & -0.22 \\
\hline 39 & $492(276-1,178)$ & 5 & $1,844(186-3,552)$ & 6 & $1,332(230-5,080)$ & 7 & 0.465 & -0.21 \\
\hline 40 & $758(330-1,040)$ & 44 & $1,512(228-3,332)$ & 4 & $1,361(245-3,279)$ & 6 & 0.176 & -0.25 \\
\hline
\end{tabular}

Data are presented as median ( $95 \%$ confidence interval for median) VEGF in pg/ml. $P$ values $<0.05$ are marked in bold.

PMA, postmenstrual age; ROP, retinopathy of prematurity; VEGF, vascular endothelial growth factor. 
a

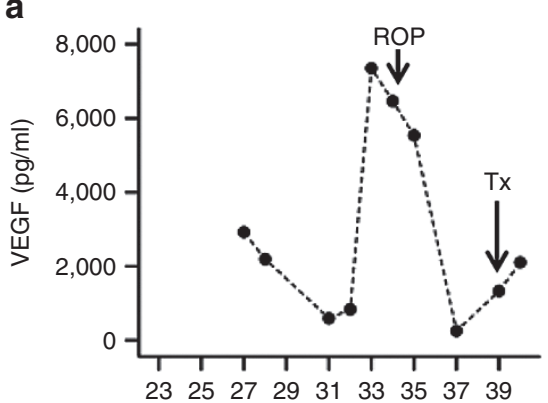

d

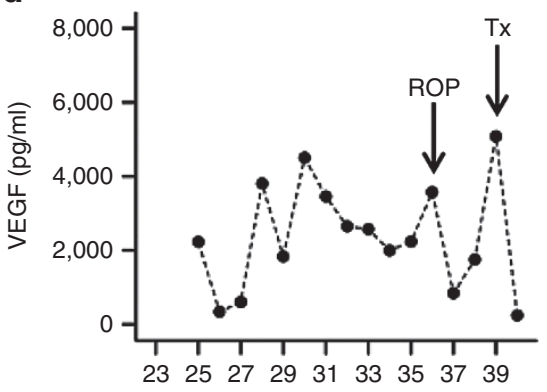

g

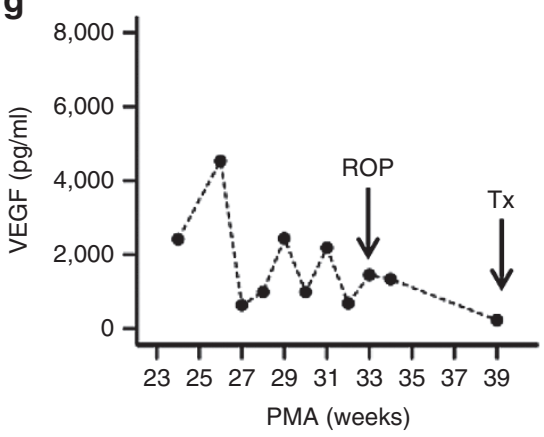

b

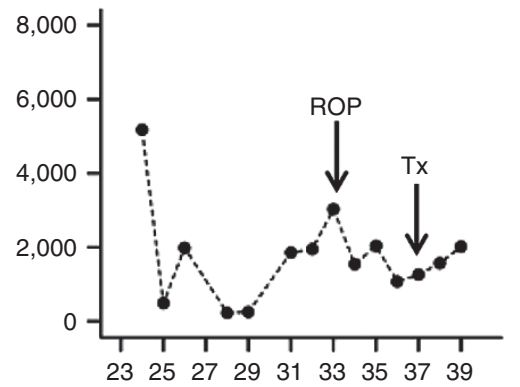

e

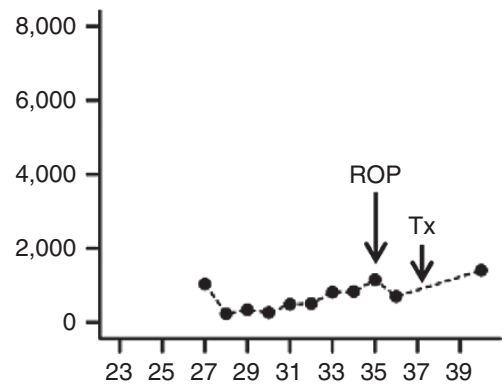

h

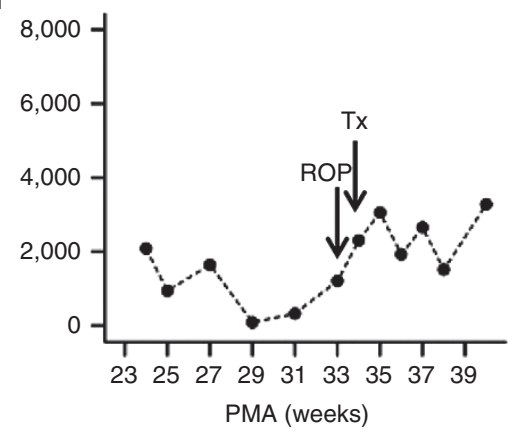

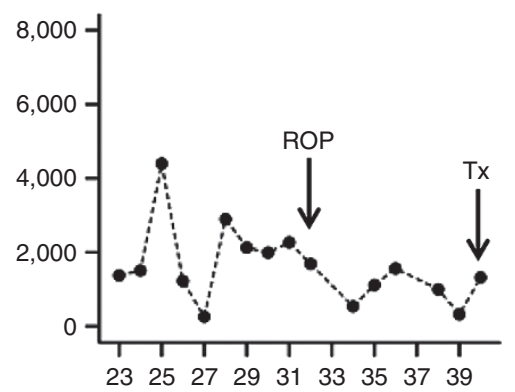

f

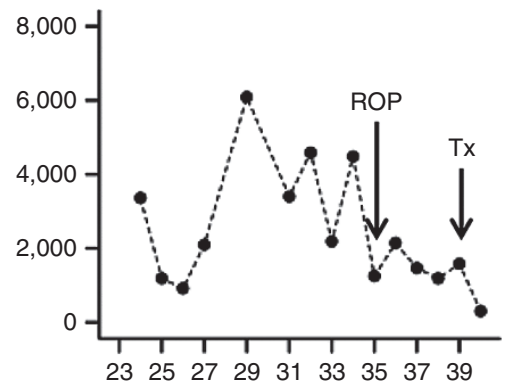

i

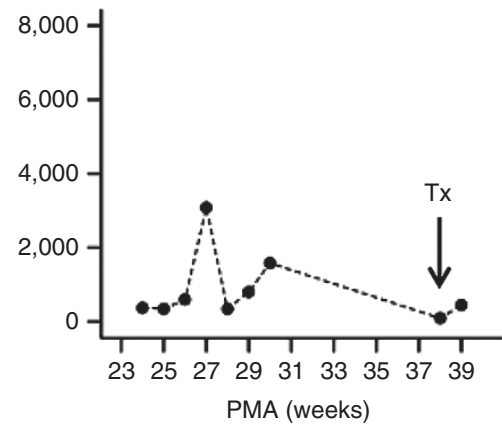

Figure 3. VEGF serum concentrations for each individual infant treated for ROP. Arrows indicate the time of the first sign of ROP and the time of laser therapy (Tx). ROP, retinopathy of prematurity; VEGF, vascular endothelial growth factor.

most infants treated for ROP, showed high VEGF serum concentrations during the nonproliferative phase of ROP but not during the proliferative stage (when ROP treatment occurred).

The role of VEGF in the development of ROP has been established in a number of oxygen-induced animal models for ROP, some with a period of constant hyperoxia, followed by relative hypoxa (8) and some with fluctuating oxygen concentrations (19). In these models, in phase I of ROP, VEGF mRNA expression is decreased in the retina with constant exposure to hyperoxia, with concomitant suppression of retinal vessel growth (20), whereas with exposure to oxygen fluctuations, increased retinal VEGF mRNA expression was seen at the stage when retinal vascularization still was incomplete and before the peak of pathologic vasoproliferation $(5,21)$. These findings together with our data support that the proliferative phase of ROP may be initiated earlier than was previously thought and that antiangiogenic or angiogenic factors other than VEGF may be important players during the vasoproliferative phase $(5,21)$. Increased understanding of growth factor regulation in relation to timing of ROP progression can be useful in predicting outcomes and optimal timing of treatment.
It is not possible to draw longitudinal vitreous samples for VEGF to correspond to serum VEGF measurements. Therefore, the biological relevance of systemic VEGF concentrations for the development of ROP is speculative. However, blood drawing is a convenient way to examine VEGF longitudinal data and is likely to reflect local levels to some degree since we know that anti-VEGF antibody injected locally can be measured systemically for months (16). We believe that the results of the present study add important information to our understanding of the biology underpinning the development of ROP.

Pieh et al. investigated longitudinal VEGF concentrations in pooled plasma samples from 63 infants of GA 23-32 wk (11). In contrast with our results, no substantial differences in VEGF concentrations between patients with ROP and without ROP or over time were found (11). In the same study, a subgroup of infants was analyzed for VEGF plasma concentrations at PMA 32 and $36 \mathrm{wk}$; no differences were detected between infants with and without later development of ROP. Here, longitudinal VEGF protein patterns were similar in all groups until PMA 34 wk. From this time point until PMA 36 wk, infants 
with proliferative ROP had higher VEGF concentrations than infants without ROP and with nonproliferative ROP. However, there are differences between these two studies. In the present study, all values were obtained from individual samples that were collected weekly, and infants were divided into three groups (non-ROP, nonproliferative ROP, and proliferative ROP). In the study by Pieh et al. (11), two to five samples taken within a mean of $7 \mathrm{~d}$ were pooled before analysis, and the infants were categorized as no ROP or ROP, which may explain the inconsistency in results.

This study has some limitations. First, VEGF serum concentration in cord blood and postnatal concentrations from the third postnatal day were analyzed using different Luminex assays. Both assays were from Bio-Rad, but the included analytes differed between assays, and hence the reagents came from different batches. The different analyte setups yielded different background values between assays, which had a substantial impact on absolute values. For multiplex assays, it is recommended that all analyses be run using the same batches of antibodies and beads and that all preanalytical and analytical conditions remain constant throughout all analyses, which could not be done in this study. As a consequence, VEGF concentrations in cord blood were not included in our longitudinal analyses and were not compared as absolute values with postnatal samples. Second, serum concentrations of VEGF reflect not just circulating VEGF but also VEGF from platelets. A different correlation and less scatter of values may have resulted from measurements of plasma VEGF concentrations. Third, this study was small, with few infants developing ROP. Our results therefore need to be validated in larger study groups. An ongoing clinical trial (CARE-ROP, ClinicalTrials. gov Identifier: NCT02134457) is addressing the relationship between VEGF concentrations and ROP status.

The main finding in this study is that longitudinal VEGF concentrations in the circulation differ at specific periods according to ROP status. In this pilot study, we found elevated VEGF concentrations in infants treated for ROP, at PMA 34$36 \mathrm{wk}$, a period corresponding to the development of ROP, but not at the later periods during which ROP treatment usually takes place.

\section{METHODS}

\section{Ethical Considerations}

The study protocol was approved by the Regional Ethical Review Board in Lund, Sweden, and the study was performed in accordance with the Declaration of Helsinki. Written informed consent was obtained from all parents.

\section{Study Population}

This study was conducted as a prospective longitudinal cohort study in infants born at Lund University Hospital, Lund, Sweden, between January 2005 and May 2007, which previously has been described in detail (22). Pregnant women admitted to Lund University Hospital with a risk of delivery before 31 gestational weeks were identified and enrolled before delivery after written informed consent from both parents were obtained. All pregnancies were dated by ultrasound at 17-18 gestational weeks. Inclusion criteria were a GA $<31$ wk at birth, antenatal informed consent from both parents, and absence from major congenital anomalies. Sixty-four infants were enrolled in the study. Twelve infants did not complete the study, nine infants died, and three parents of infants declined continued participation, leaving 52 infants for evaluation of longitudinal serum concentrations.

Neonatal data on gender, GA at birth, birth weight, Apgar score, patent ductus arteriosus (requiring medical or surgery treatment), bronchopulmonary dysplasia, necrotizing enterocolitis, and intraventricular hemorrhage were obtained from the infant's records. bronchopulmonary dysplasia was defined as need of supplemental oxygen at a PMA of $36 \mathrm{wk}$. Severe intraventricular hemorrhage was defined in the presence of intraventricular hemorrhage grade 3 and/or parenchymal hemorrhagic infarction, and necrotizing enterocolitis was defined by clinical and radiological criteria.

\section{Growth Measurements}

Standardized measurements of weight were performed within $24 \mathrm{~h}$ after birth. Standard deviation score for weight (weight $t_{\text {cDs }}$ ) was calculated from an intrauterine growth curve based on ultrasonically estimated fetal weights in Scandinavia (23).

\section{ROP Examinations}

ROP was classified according to the International Classification (24) and subdivided into stage 1 (demarcation line), stage 2 (ridge), stage 3 (ridge with extraretinal fibrovascular proliferation), stage 4 (subtotal retinal detachment), and stage 5 (total retinal detachment). Each child was classified according to the most advanced ROP stage observed. Proliferative retinopathy was defined as ROP stage $\geq 3$ and nonproliferative ROP as stages 1 and 2. The infants were examined according to a routine protocol that consisted of dilated eye fundus examinations once or twice per week, depending on the severity of the disease, from the chronological age of 5-6wk until retinas were fully vascularized. After pupillary dilatation with $1 \%$ cyclopentolate, the eyes were examined via indirect ophthalmoscopy by a trained pediatric ophthalmologist. Thirty-three infants were classified as non-ROP, 10 infants were classified as having nonproliferative ROP, and 9 infants were classified as having proliferative ROP and were later treated with laser for ROP. The international recommendation was used for ROP treatment and was based on the following criteria: the stage of ROP, the zone in which ROP was detected, and whether plus disease was detected (25). All treated children had ROP stage 3 (zone II and III) with plus disease.

\section{Blood Sampling and Quantitative VEGF Analyses}

At birth, cord blood plasma was collected from 49 infants. For longitudinal analyses, blood serum sampling was performed before enteral feeding from 52 infants at $72 \mathrm{~h}$ and at $7 \mathrm{~d}$ postnatal age and thereafter weekly until at least a PMA of $35 \mathrm{wk}$. Complete data, i.e., cord plasma samples and longitudinal serum samples for analyses of VEGF, and ROP data were available for 39 infants. After centrifugation, samples were stored at $-80^{\circ} \mathrm{C}$ until assayed. All samples were diluted 1:4 before analysis.

VEGF plasma concentrations in cord blood were measured in duplicate using a human Luminex xMAP assay including VEGF (BioRad Laboratories, Hercules, CA) according to the manufacturer's protocol. The lowest level of quantification was $1.88 \mathrm{pg} / \mathrm{ml}$. Samples under the quantification limit were set to half this value $(0.94 \mathrm{pg} / \mathrm{ml})$. The inter-assay coefficient of variation was $15.05 \%$ at $38.18 \mathrm{pg} / \mathrm{ml}$. The mean intra-assay coefficient of variation between duplicates was $5.47 \%$. Beads and antibodies within the assay were from the same batches, and all plates were run by the same operator.

Serum from blood samples collected from $3 \mathrm{~d}$ postnatal age and forward was assayed with another human Luminex xMAP assay including VEGF (Bio-Rad Laboratories). The lowest level of quantification was $1.93 \mathrm{pg} / \mathrm{ml}$. The inter-assay coefficient of variation for VEGF was $14.36 \%$ at $291 \mathrm{pg} / \mathrm{ml}$. An internal control sample was analyzed in 2-10 replicates in each run with a mean intra-assay coefficient of variation of $9.98 \%$. Beads and antibodies within the assay were from the same batches, and all but three plates were run by the same operator. Luminex multiplex assays only detect free VEGF.

\section{Statistical Analysis}

Statistical analyses were performed using the program package IBM SPSS Statistics 20 for Microsoft Windows (IBM, Armonk, NY). Kruskal-Wallis or chi-squared tests were used for analyses of more than two groups. The Mann-Whitney $U$-test was used for analyses of 
independent groups, and the Wilcoxon signed rank test was used for analyses of two paired samples. Effect size was calculated by Cohen's $d$. Spearman rank order test was used for correlation analyses. Cord blood samples were not included in longitudinal data analyses after birth as cord blood analyses were performed on plasma samples, and longitudinal analyses were performed on serum samples. In addition, a substantial number of cord blood samples were missing, and cord blood samples and samples collected after birth were analyzed with different assay setups. $P$ values $<0.05$ were considered significant for all analyses.

\section{STATEMENT OF FINANCIAL SUPPORT}

This study has been supported by the Swedish Medical Research Council (2011-2432, 2011-3893), Stockholm, Sweden; a Swedish government grant (ALFGB2770), Gothenburg, Sweden; Lund medical faculty grants (ALFLund11615 and 11601), Lund, Sweden; the Skåne Council Foundation for Research and Development, Lund, Sweden; the Linnéa and Josef Carlsson Foundation, Helsingborg, Sweden; De Blindas Vänner, Gothenburg, Sweden; and Crown Princess Margareta's Foundation for Visual Impairment, Valdermarsvik, Sweden.

Disclosure: The authors have no financial relationships relevant to this article to disclose.

\section{REFERENCES}

1. Hughes S, Yang H, Chan-Ling T. Vascularization of the human fetal retina: roles of vasculogenesis and angiogenesis. Invest Ophthalmol Vis Sci 2000;41:1217-28.

2. Hartnett ME. Pathophysiology and mechanisms of severe retinopathy of prematurity. Ophthalmology 2015;122:200-10.

3. Hartnett ME, Penn JS. Mechanisms and management of retinopathy of prematurity. N Engl J Med 2012;367:2515-26.

4. Smith LE. Through the eyes of a child: understanding retinopathy through ROP the Friedenwald lecture. Invest Ophthalmol Vis Sci 2008;49:5177-82.

5. Werdich XQ, McCollum GW, Rajaratnam VS, Penn JS. Variable oxygen and retinal VEGF levels: correlation with incidence and severity of pathology in a rat model of oxygen-induced retinopathy. Exp Eye Res 2004;79:623-30.

6. Austeng D, Källen KB, Hellström A, Tornqvist K, Holmström GE. Natural history of retinopathy of prematurity in infants born before 27 weeks' gestation in Sweden. Arch Ophthalmol 2010;128:1289-94.

7. Pierce EA, Avery RL, Foley ED, Aiello LP, Smith LE. Vascular endothelial growth factor/vascular permeability factor expression in a mouse model of retinal neovascularization. Proc Natl Acad Sci USA 1995;92:905-9.

8. Smith LE, Wesolowski E, McLellan A, et al. Oxygen-induced retinopathy in the mouse. Invest Ophthalmol Vis Sci 1994;35:101-11.

9. Galazios G, Papazoglou D, Giagloglou K, Vassaras G, Koutlaki N, Maltezos E. Umbilical cord serum vascular endothelial growth factor (VEGF) levels in normal pregnancies and in pregnancies complicated by preterm delivery or pre-eclampsia. Int J Gynaecol Obstet 2004;85:6-11.

10. Woo SJ, Park KH, Lee SY, et al. The relationship between cord blood cytokine levels and perinatal factors and retinopathy of prematurity: a gestational age-matched case-control study. Invest Ophthalmol Vis Sci 2013;54:3434-9.

11. Pieh C, Agostini H, Buschbeck C, et al. VEGF-A, VEGFR-1, VEGFR-2 and Tie2 levels in plasma of premature infants: relationship to retinopathy of prematurity. Br J Ophthalmol 2008;92:689-93.

12. Malamitsi-Puchner A, Tziotis J, Tsonou A, Protonotariou E, Sarandakou A, Creatsas G. Changes in serum levels of vascular endothelial growth factor in males and females throughout life. J Soc Gynecol Investig 2000;7: $309-12$.

13. Sato T, Kusaka S, Shimojo H, Fujikado T. Simultaneous analyses of vitreous levels of 27 cytokines in eyes with retinopathy of prematurity. Ophthalmology 2009;116:2165-9.

14. Sonmez K, Drenser KA, Capone A Jr, Trese MT. Vitreous levels of stromal cell-derived factor 1 and vascular endothelial growth factor in patients with retinopathy of prematurity. Ophthalmology 2008;115: 1065-1070.e1.

15. Ma J, Mehta M, Lam G, et al. Influence of subretinal fluid in advanced stage retinopathy of prematurity on proangiogenic response and cell proliferation. Mol Vis 2014;20:881-93.

16. Kong L, Bhatt AR, Demny AB, et al. Pharmacokinetics of bevacizumab and its effects on serum VEGF and IGF-1 in infants with retinopathy of prematurity. Invest Ophthalmol Vis Sci 2015;56:956-61.

17. Sato T, Wada K, Arahori H, et al. Serum concentrations of bevacizumab (avastin) and vascular endothelial growth factor in infants with retinopathy of prematurity. Am J Ophthalmol 2012;153:327-333.e1.

18. Yalin Imamoglu E, Gunay M, Gursoy T, et al. Effect of laser photocoagulation on plasma levels of VEGF-A, VEGFR-2, and Tie2 in infants with retinopathy of prematurity. J AAPOS 2014;18:466-70.

19. Penn JS, Henry MM, Tolman BL. Exposure to alternating hypoxia and hyperoxia causes severe proliferative retinopathy in the newborn rat. Pediatr Res 1994;36:724-31.

20. Pierce EA, Foley ED, Smith LE. Regulation of vascular endothelial growth factor by oxygen in a model of retinopathy of prematurity. Arch Ophthalmol 1996;114:1219-28.

21. Budd SJ, Hartnett ME. Increased angiogenic factors associated with peripheral avascular retina and intravitreous neovascularization: a model of retinopathy of prematurity. Arch Ophthalmol 2010;128: 589-95.

22. Hansen-Pupp I, Löfqvist C, Polberger S, et al. Influence of insulin-like growth factor I and nutrition during phases of postnatal growth in very preterm infants. Pediatr Res 2011;69:448-53.

23. Marsál K, Persson PH, Larsen T, Lilja H, Selbing A, Sultan B. Intrauterine growth curves based on ultrasonically estimated foetal weights. Acta Paediatr 1996;85:843-8.

24. An international classification of retinopathy of prematurity. The committee for the classification of retinopathy of prematurity. Arch Ophthalmol 1984;102:1130-4.

25. Good WV, Hardy RJ, Dobson V, et al.; Early Treatment for Retinopathy of Prematurity Cooperative Group. The incidence and course of retinopathy of prematurity: findings from the early treatment for retinopathy of prematurity study. Pediatrics 2005;116:15-23. 